\title{
Revista Colombiana de

\section{Capítulo 2. Introducción, epidemiología de la falla cardiaca e historia de las clínicas de falla cardiaca en Colombia}

\section{Efraín Gómez}

\author{
Cardiología, Clínica Shaio. Bogotá, Colombia
}

Recibido el 17 de noviembre de 2015; aceptado el 12 de enero de 2016

Disponible en Internet el 5 de febrero de 2016

\section{PALABRAS CLAVE Insuficiencia \\ cardiaca; \\ Estudios de seguimiento; Pronóstico}

\section{KEYWORDS}

Heart failure; Follow-up studies;

Prognosis

\begin{abstract}
Resumen
Introducción: La edad de la población, tanto mundial como colombiana, y la prolongación de la vida con reducción de la mortalidad de los pacientes cardiacos, han llevado a un incremento de la falla cardiaca. Durante los últimos diez años se han creado varias clínicas de falla cardiaca y de esta manera se ha logrado avanzar en las alternativas de tratamiento para la enfermedad. Objetivo: Describir las características de las diferentes clínicas de falla cardiaca que funcionan actualmente en Colombia.

Metodología: Se realizó una revisión narrativa de la información sobre los recursos y servicios disponibles en cada centro de Colombia.

Conclusión: Las clínicas de falla cardiaca surgieron en Colombia en 1985. Desde entonces se cuenta con varios programas en ciudades como Bogotá, Medellín, Cali y Bucaramanga que ofrecen la opción de seguimiento multidisciplinario por Enfermería y Cardiología, e incluso muchas de ellas tienen programas de trasplante de corazón. Sin embargo, el reto actual para todos los programas es continuar creciendo e integrándose con los niveles básicos de atención para permitir intervenir desde la prevención, el diagnóstico temprano y el tratamiento adecuado de la falla cardiaca.

๑) 2016 Publicado por Elsevier España, S.L.U. en nombre de Sociedad Colombiana de Cardiología y Cirugía Cardiovascular. Este es un artículo Open Access bajo la licencia CC BY-NC-ND (http://creativecommons.org/licenses/by-nc-nd/4.0/).
\end{abstract}

Introduction, epidemiology of heart failure and the history of cardiac failure clinics in Colombia

\section{Abstract}

Introduction: The age of both world and Columbian populations, and extended life-times along with a reduction in the mortality of cardiac patients has resulted in an increase in heart failure. 
Several heart failure clinics have been set up over the last ten years and thus advances have been made in the treatment alternatives for this disease.

Objetive: To describe the characteristics of the different heart failure clinics which are currently in operation in Colombia.

Methodology: A narrative review of the information available on the available resources and services in each centre in Colombia.

Conclusion: Heart failure clinics came into being in Colombia in 1985. Since then there are various programmes in cities such as Bogota, Medellin, Cali and Bucaramanga which offer the option of multidisciplinary follow-up by nursing and cardiology staff, and many of them even have heart transplantation programmes. However, the current challenge facing all of these programmes is to continue to grow and integrate with the basic levels of care in order to act in the prevention, early diagnosis and appropriate treatment of heart failure.

(c) 2016 Published by Elsevier España, S.L.U. on behalf of Sociedad Colombiana de Cardiología y Cirugía Cardiovascular. This is an open access article under the CC BY-NC-ND license (http://creativecommons.org/licenses/by-nc-nd/4.0/).

\section{Introducción}

La edad de la población, tanto mundial como colombiana, y la prolongación de la vida con reducción de la mortalidad de los pacientes cardiacos han causado un incremento de la falla cardiaca. Pese a los grandes avances terapéuticos en este campo, la mortalidad por esta condición sigue siendo inaceptablemente alta.

\section{Prevalencia}

Una actualización de 2013 de la American Heart Association (AHA, su sigla en inglés) estimó que en 2006, 5.1 millones de personas padecieron falla cardiaca en los Estados Unidos ${ }^{1}$ y que en el mundo fueron 23 millones las afectadas por este flagelo ${ }^{2}$.

Para Colombia, con una población de 47.704.472 habitantes, la prevalencia de falla cardiaca estimada es del 2,3\%, lo que se traduce en cerca de 1.097.201 pacientes con falla cardiaca en el país.

En la figura 1 se observa la etiología de los pacientes tratados en seis clínicas de falla cardiaca de Colombia, con una muestra de 4.239 casos en la que se aprecia que la etiología número 1 es la enfermedad cardiaca isquémica seguida de la idiopática y en tercer lugar la enfermedad cardiaca valvular y la de Chagas.

\section{Incidencia}

Al igual que la prevalencia, la incidencia de falla cardiaca va aumentando con la edad. En el estudio de Framinghan, la incidencia se duplicó aproximadamente por cada década de la vida, incrementando su porcentaje con la edad más en la mujer que en el hombre.

La incidencia en estos últimos aumentó de 2 por 1.000 a la edad de 35 a 64 años, a 12 por 1.000 a la edad de 65 a 94 años.

En la figura 2 se evidencia la tasa de mortalidad por falla cardiaca en Colombia según datos del Sistema de

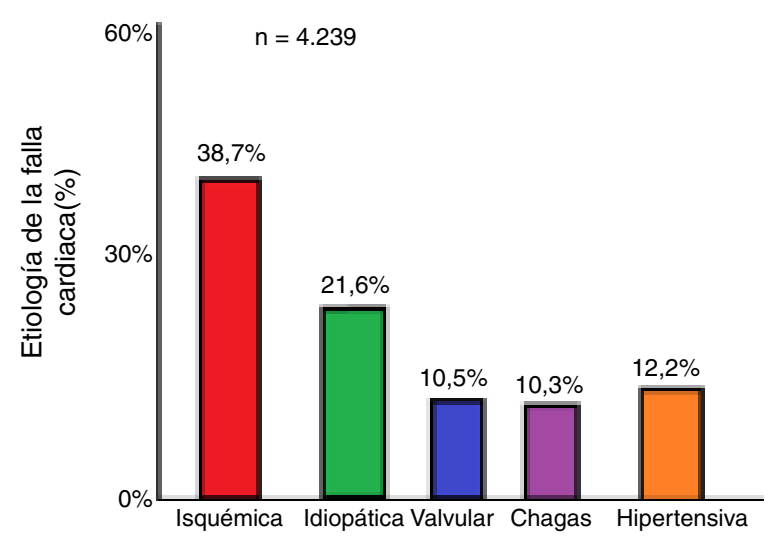

Figura 1 Etiología de la falla cardiaca.

información de la protección social, que fue del 5,54 por 100.000 habitantes para el año 2012.

\section{Historia de las clínicas de falla cardiaca en Colombia}

En 1995, la Clínica Shaio de Bogotá, en conjunto con el proceso de implementación del programa de trasplantes de corazón de esa institución, decidió crear la primera clínica de falla cardiaca del país, con un programa que inició bajo el liderazgo del médico cardiólogo el Dr. Efraín Gómez y la Jefe de enfermería Liliana Ferro, dedicada de tiempo completo al programa de educación y al seguimiento de pacientes. Se inscribieron cerca de 50 pacientes con falla cardiaca avanzada en un programa estructurado de manejo y seguimiento que permitiera disminuir rehospitalizaciones y síntomas, y ayudara a mejorar la de calidad de vida, y que adicionalmente permitiera trasladarlos al programa de trasplantes en caso de persistencia de síntomas y clase funcional avanzada. En la actualidad la clínica de falla cardiaca cuenta con cuatro cardiólogos clínicos (los Drs. Fernán Mendoza actual jefe del programa, Claudia Jaramillo, Marcela Poveda y Efraín Gómez), y la Jefe de enfermería Silvia Martínez), 


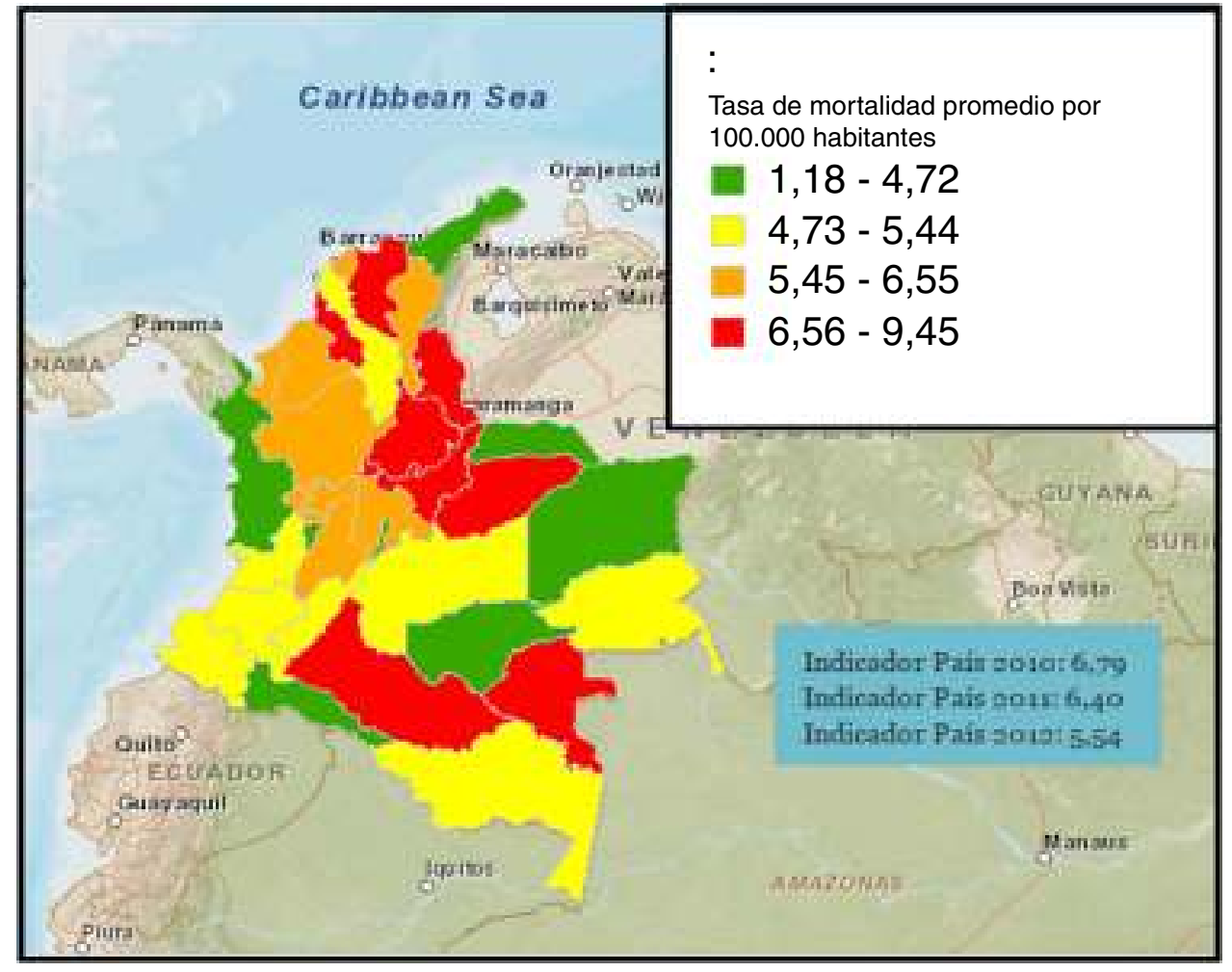

Figura 2 Tasa de mortalidad promedio por 100.000 habitantes en Colombia.

todas las especialidades de apoyo y un programa de hospital día y de manejo inotrópico intermitente que comenzó hace aproximadamente un año.

Una década atrás, en 1985, se realizaba en Medellín el primer trasplante de corazón en la Clínica cardiovascular Santa María, institución pionera en este tipo de intervenciones y ejemplo y ayuda para el desarrollo de otros programas del país, como fue el caso del programa de trasplantes de la Clínica Shaio, para el cual se contó con la valiosa asesoría del Dr. Carlos Tenorio, fundamental para el inicio del programa, en cabeza de los Doctores Efraín Gómez, del área de falla cardiaca y trasplantes, y Juan Rafael Correa, del segmento de cirugía cardiovascular.

Fue entonces que en 1996 se hizo el primer trasplante de corazón en la Clínica Shaio, y el segundo en Bogotá, pues la Fundación Santa Fe había efectuado el primero en la ciudad dos años antes.

Con la activación de los programas de trasplante en Bogotá y otras ciudades como Cali y Bucaramanga, el Estado y las instituciones trasplantadoras de Colombia (en esa época 6 en total), decidieron crear la Comisión nacional de corazón, que organizaba las estrategias para el manejo de los trasplantes de corazón y otros órganos en el país.

Para 1996 y durante la presidencia del Dr. Adolfo Vera, se logró la creación del Consejo nacional de falla cardiaca en cabeza del Dr. Efraín Gómez con miras a aglutinar colegas cardiólogos interesados en el manejo de pacientes con falla cardiaca y motivar la creación de programas de educación médica continuada sobre este tópico en diferentes escenarios clínicos a fin de mejorar el nivel de manejo de pacientes con esta patología.
A través del consejo se logró la producción de la primera "Guía colombiana de manejo de pacientes con falla cardiaca', publicada en 2007.

La coordinación de este consejo fue precedida por el Dr. Solón Navarrete y fue así como el grupo de colegas interesados en falla cardiaca fue ampliándose y durante la dirección de la Dra. Adriana Torres en cumplimiento de los requisitos para la conformación del consejo, nació el Capítulo de Falla Cardiaca (año 2012), el cual ha sido a la fecha uno de los más productivos en temas de educación médica continuada de la Sociedad Colombiana de Cardiología y Cirugía Cardiovascular.

En 2013 y con el apoyo de la Sociedad Española de Cardiología, bajo el mando del Dr. Josep Comin-Collet del Hospital de Mar en Barcelona y en asocio con el Capítulo de falla cardiaca de la Sociedad Colombiana de Cardiología y Cirugía Cardiovascular, liderado por la Dra. Adriana Torres, se llevó a cabo el primer curso itinerante para desarrollar e implementar nuevos programas de clínicas de falla cardiaca en Colombia. El proyecto se replicó con la participación exclusiva de los miembros del capítulo 2015, invitándose a colegas de otros países a desarrollar programas de falla cardiaca, bajo la dirección de la Dra. Clara Inés Saldarriaga, actual presidenta del Capítulo, quien ha seguido con entusiasmo los programas itinerantes de educación médica continuada en falla cardiaca por todo el país, y ha tenido avances importantes como los consensos nacionales de falla cardiaca crónica y falla cardiaca aguda y la participación de algunos de sus miembros en representación de la Sociedad Colombiana de Cardiología en la primera "Guía colombiana de falla cardiaca crónica"' en consorcio con las universidades de Antioquia, Nacional y Pontificia Javeriana, y otras 
sociedades científicas como la Sociedad colombiana de Medicina Interna, con el soporte del Ministerio de Salud de Colombia, convirtiéndose en ejemplo para Latinoamérica en el diseño de este tipo de guías en las que se hace análisis de costo-efectividad para el país en muchas de sus estrategias terapéuticas.

De otro lado, también para 1996 en la Fundación Valle de Lili en Cali, se realizó el primer trasplante cardiaco e inició el programa de falla cardiaca con el Dr. Noel Alberto Flórez. Al año siguiente se incorporó al programa el Dr. Pastor Olaya y juntos se encargan del manejo de los pacientes ambulatorios y hospitalizados de falla cardiaca y de todo lo relacionado con el programa de trasplantes. Posteriormente, en 2002, ambos colegas se actualizaron y ampliaron conocimientos en estas áreas en los Estados Unidos. En 2006 se incorporó al servicio el doctor Juan Esteban Gómez, quien regresó de su formación en Falla Cardiaca en New Orleans, Estados Unidos. Luego de su ingreso se inició también la atención de los pacientes con hipertensión pulmonar en el mismo servicio. Para 2008 se logró la vinculación de una enfermera profesional de medio tiempo (Bibiana Mesa), quien laboró en el servicio hasta 2009, y a partir de esa fecha ingresó la enfermera Julia Arango, a quien le sucedió en su cargo la Enfermera Lucy Rivera, vinculada tiempo completo a partir de noviembre de 2015.

En la actualidad se atienden cada mes aproximadamente 200 pacientes ambulatorios y un promedio de 40 pacientes hospitalizados con falla cardiaca e hipertensión pulmonar, así como todos los servicios incluidos dentro del programa de trasplante cardiaco. Se han realizado 170 trasplantes de corazón y a partir de este año se inició el programa de trasplante de pulmón, con el cual también se podrán beneficiar algunos pacientes con hipertensión arterial pulmonar. Dentro de otros servicios especiales están hospital día, manejo ambulatorio de infusiones, cuidado domiciliario (Home Care), además de la atención y servicio multidisciplinario que se puede ofrecer dentro de la Fundación Valle del Lili.

Así mismo, para el año 2000 en la Fundación Cardioinfantil se inició una consulta de atención externa preferencial para pacientes con falla cardiaca y se hizo un acuerdo con todo el grupo de cardiólogos de la institución para derivar a ésta los pacientes con diagnóstico de falla cardiaca y dar paso a un ciclo de atención integral con apego a las guías clínicas de manejo.

Se iniciaron estudios de profundización en falla y trasplante cardiaco, gracias a los convenios con la Cleveland Clinic de Ohio, Estados Unidos y el Hospital 12 de Octubre de Madrid, España.

El 10 de mayo de 2005 la dirección de la fundación y la oficina de planeación aprobaron la creación formal de la clínica de falla cardiaca y se estableció como plataforma para la implementación del programa de trasplante de corazón. Inicialmente, las actividades se desarrollaron en los consultorios de la consulta externa asignados para las actividades de cardiología clínica, y así mismo se eligió un modelo de funcionamiento basado en la coordinación de una jefe de enfermería respaldada por dos médicos cardiólogos, con entrenamiento en falla cardiaca. Se comenzó la aplicación del modelo de atención y cuidado con 120 pacientes inscritos, bajo la coordinación de la jefe de enfermería María Teresa Ibarra y los médicos cardiólogos Mónica López y Camilo Franco. Se implementaron las guías de autocuidado del paciente y se imprimieron volantes educativos y de promoción a fin de dar inicio a los ciclos de educación al paciente y el grupo familiar, del cual, a agosto de 2015, se han efectuado 8.466 sesiones de educación.

Se creó una base de datos donde se consignan entre otros datos, tipo de cardiopatía, fracción de eyección, clase funcional, intervenciones, medicamentos, vacunación, etc. que facilitan el seguimiento de los pacientes.

Para agosto de 2005 se completó la estructuración de la "Guía clínica para el Trasplante de Corazón", con la participación de los servicios de Falla cardiaca bajo el mando de los Drs. Mónica López y Camilo Franco, Cirugía cardiovascular en cabeza del Dr. Javier Maldonado, Anestesia con los Drs. Hernán Charris y Juan Camilo Giraldo, Infectología con Dr. Álvaro Arango, Rehabilitación Cardiovascular con la Dra. Astrid Fajardo, Trabajo Social con Fabiola Villalba, Unidad de Cuidado Quirúrgico con el Dr. Daniel Isaza y Salud Mental con los Drs. Manuel Torres y María Teresa Gómez.

Fue así como el 4 de noviembre de 2005 se llevó a cabo allí el primer trasplante de corazón.

Entre tanto, para el año 2014, bajo la dirección científica del Dr. Juan Pablo Umaña se lideró un proceso de crecimiento del servicio de falla cardiaca, se vincularon al grupo los Drs. Adriana Torres y John Ramírez y se nombraron en exclusividad para el servicio la Nutricionista Johana Rojas, la Trabajadora Social Sandra Herrera, las Enfermeras Jefes María Fernanda Tamayo y Sandra Leonor Molina, la Auxiliar Angee Paola Ballesteros, y la Auxiliar administrativa Helena Acosta.

En la actualidad se desarrolla un programa de Telemedicina y se ha comenzado con éxito el programa de Asistencia Circulatoria Mecánica. Se construyó la Unidad de Falla Cardiaca / Hospital de día, la cual entregó a finales de 2015 este servicio dirigido a atender la demanda de pacientes ambulatorios que requieren intervenciones como aplicación de diuréticos, inotrópicos o hierro parenteral, y además permitirá disminuir el número de hospitalizaciones y la ocupación de los servicios de urgencias.

Hoy día, la unidad de falla Cardiaca cuenta con 400 pacientes inscritos, adheridos, y en seguimiento agrupa 69 pacientes beneficiados con trasplantes cardiaco, de los cuales 10 se atendieron en el último año.

Para el año 2000 en el Hospital Central de la Policía en Bogotá y bajo la dirección del Dr. Solón Navarrete se inició la clínica de falla cardiaca. En 2005, se creó una alianza con el programa de Rehabilitación cardiovascular y el grupo de Electrofisiología de la misma institución. Posteriormente, se pusieron en marcha los programas de hospital día de los pacientes con falla cardiaca, y adicionalmente crearon una clínica de pacientes con enfermedad de Chagas.

En 2002, se dio inicio al programa de falla cardiaca en la Fundación Cardiovascular de Colombia en Bucaramanga, a cargo del Dr. Manuel Guillermo Hernández, en conjunto con una enfermera, convirtiéndose así en la primera clínica de falla cardiaca del nororiente colombiano.

El primer año incluyó 80 pacientes valorados en hospitalización y consulta ambulatoria, y en 2004 se hizo el primer trasplante cardíaco en esa institución y el primero en Santander.

En 2005 fue nombrado como director del programa de falla cardiaca y trasplantes el actual director Dr. Luis 
Eduardo Echeverría. En este tiempo se han atendido 1.721 pacientes con más de 700 pacientes activos a la fecha y se han realizado 106 trasplantes cardiacos. Fue el primer grupo en Colombia y Suramérica en colocar la primera asistencia ventricular (2014), y al momento trata tres pacientes con asistencias ventriculares a largo término tipo HeartMate $॥$.

En 2014 se inició el programa de hospitalización día para administración de inotrópicos intermitentes, anemia cardiorrenal y diuréticos endovenosos. Hoy, el servicio de falla cardiaca de la Fundación Cardiovascular, cuenta con los cardiólogos, Drs. María Juliana Rodríguez y Jaime Alberto Rodríguez, la enfermera jefe Adriana Jurado, una psicóloga, una nutricionista, una fisioterapeuta y una auxiliar de enfermería, además de la colaboración del servicio de Rehabilitación cardiaca bajo la tutela de la Dra. Laura Báez.

Hacia el año 2003 en el Hospital San Ignacio de Bogotá, se instauró el programa de trasplante cardiaco, pero ya que aun no contaba con una clínica de falla cardiaca decidieron enviar a entrenamiento en trasplante cardiaco a Barcelona a los cardiólogos: los Drs. Alex Rivera y Javier Beltrán, así como a la Dra. Nubia Lucía Roa a Baltimore, para una rotación en falla cardiaca con el Dr. Steve Gotlieb en la Universidad de Maryland.

Luego de esto se constituyó el programa de falla cardiaca en 2004, que inicialmente no contó con la presencia de enfermería, pero se abriera un cargo en propiedad en el año 2006 (Luisa Fernanda Aponte) bajo la dirección del Dr. Jaime Alvarado del Departamento de Medicina Interna y el Dr. Ricardo Bohórquez de la Unidad de Cardiología, asignándose además un espacio para la Unidad de Falla cardiaca.

El equipo inicial de los tres cardiólogos trató a los pacientes de trasplante y falla cardiaca en compañía de los cirujanos que conformaban el equipo del programa de trasplante cardiaco, los Drs. Juan Rafael Correa, quien años atrás perteneciera a la Clínica Shaio y fuera uno de los fundadores del programa de trasplantes y Federico Núñez quien hoy día es cirujano cardiovascular del programa de trasplantes de la Clínica Shaio.

El Dr. Alex Rivera dejó el Hospital San Ignacio en el año 2006 y la Dra. Nubia Lucía Roa fue enviada a Madrid (España) al Hospital Puerta de Hierro para entrenarse en trasplante cardiaco. En la actualidad es la directora del programa del Hospital San Ignacio.

Para octubre de 2015 estuvieron activos 279 pacientes y realizaron 46 trasplantes de corazón con el apoyo de todas las subespecializadas necesarias para el soporte del programa de falla cardiaca y trasplantes.

Desde el mes de abril 2015 se vinculó en apoyo al programa de falla cardiaca el Dr. Alejandro Mariño.

Por su parte, en 2008 se inició el programa de falla cardiaca de la Clínica Universitaria Colombia en Bogotá, bajo la dirección de la Dra. Adriana Torres con la consolidación y el inicio de actividades de un grupo multidisciplinario en diciembre de ese mismo año; el modelo logró en poco tiempo tener un crecimiento importante y de gran proyección dentro de una población cautiva de la organización Sanitas Internacional, con una infraestructura que favoreció el éxito del programa, un centro de referencia institucional concentrado en la Clínica Colombia, un sistema integrado de referenciación con atención primaria y el logro del objetivo estratégico de ser una unidad funcional de excelencia institucional de alta complejidad, con proyección nacional e internacional, como modelo de gestión de calidad, crecimiento académico y de costo-efectividad en este grupo de pacientes. Posteriormente, 2012, inició el programa de trasplante cardiaco y se involucraron más profesionales cardiólogos a apoyar el programa de falla cardiaca y trasplantes y hoy está conformada por los cardiólogos Drs. Carlos Andrés Arias, actual director del programa, Jeffrey Castellanos y Paula Burbano, dos enfermeras jefes, Diana Molano y Carolina Hernández, una nutricionista, y una trabajadora social. En 2013, se dio paso a la expansión con la integración del programa de hipertensión pulmonar como condición asociada a la falla cardiaca y el ingreso de un neumólogo. Hoy se cuenta con registro de atención de 2.500 pacientes desde su inicio, 650 pacientes activos en seguimiento en el programa, 17 trasplantes y 7 casos de asistencia ventricular como puente a recuperación o trasplante. Dentro de las innovaciones y evaluando la oportunidad dentro de nuestro esquema de aseguramiento con el apoyo institucional, se dio paso a hacer realidad, bajo el contexto de la red integrada en diferentes niveles de complejidad, la unidad de atención primaria de falla cardiaca que cuenta con 850 pacientes adicionales en seguimiento en el último año, al inicio del programa de telemedicina en su fase inicial en la ciudad de Ibagué y próximamente San Andrés, y a la proyección de la creación de otra unidad de falla cardiaca nivel ॥ de complejidad en la Clínica Reina Sofía, en Bogotá.

En 2011, se creó la Unidad de insuficiencia cardiaca de DIME Clínica Neurocardiovascular, la cual fue fundada con el objetivo de mejorar el pronóstico y la calidad de vida de los pacientes con insuficiencia cardiaca, así como de reducir la frecuencia de hospitalizaciones por descompensaciones y los costos asociados al tratamiento de la enfermedad en el Valle del Cauca. La unidad de insuficiencia cardiaca ofrece una atención basada en la asistencia por parte de un equipo multidisciplinario (cardiólogo y enfermeras especializados en el tratamiento de la insuficiencia cardiovacular, nutricionista y psicóloga) que garantiza la atención integral de los pacientes y sus familias.

Esta unidad inició actividades el 1 de junio de 2011 gracias al apoyo de la alta gerencia y al deseo organizacional de ofrecer servicios innovadores y de calidad.

Desde su puesta en marcha cuenta con asistencias circulatorias mecánicas como puente a trasplante cardiaco y/o recuperación en pacientes con insuficiencia cardiaca aguda, y ha ido incorporando herramientas tecnológicas como la monitorización remota de los dispositivos de resincronización cardiaca, con el fin de detectar y tratar con prontitud las posibles causas de descompensación.

A la fecha se han atendido cerca de 200 pacientes con insuficiencia cardiaca aguda y 800 pacientes con insuficienca cardiaca crónica, de los cuales alrededor del 40\% continúan en control y seguimiento en la unidad. Las tasas de rehospitalización por descompensación posterior al alta a 60,180 y 360 días son de $10,2 \%, 5,6 \%$ y 5,6\% respectivamente. La estancia hospitalaria promedio es de 4 días y la mortalidad relacionada con progresión de la enfermedad a 60,180 y 360 días posterior al alta es de $8,4 \%, 7,5 \%$ y $6,8 \%$ respectivamente. Se han realizado 27 trasplantes cardiacos con resultados equiparables a los publicados por la Sociedad internacional para el trasplante de corazón y pulmón (ISHLT). 
Esta unidad cumple con las características organizativas (gestión por procesos) y de prestación de servicios que se consideran óptimas para el abordaje altamente especializado de pacientes con insuficiencia cardiaca, y con la certificación ISO 9001 del ICONTEC en altos estándares de calidad. Dentro este contexto de calidad, en 2014 se llevó a cabo un proceso de referenciación con la unidad de insuficiencia cardiaca de Lancaster General Hospital (Pennsylvania), que facilitó el desarrollo de planes de mejoramiento en búsqueda de la excelencia en la atención, hecho que les permitió consolidarse posteriormente como centro de referenciación para el Instituto Nacional de Salud en Tijuana (México).

En cuanto al futuro de la unidad y en concordancia con los objetivos del plan estratégico y de valores institucionales de DIME, se busca estar a la vanguardia en innovación, a través de la incorporación de nuevas tecnologías costoefectivas para el sistema, la consolidación y el fomento de la investigación, la preservación de la excelencia en la atención tanto desde el punto de vista científico como humano y el alimento del espíritu profesional de todos los que allí trabajan.

En 2012, la clínica CardioVID de Medellín, centro especializado de cuarto nivel de complejidad, líder en el tratamiento de pacientes con enfermedades cardiovasculares, neurovasculares y pulmonares en Colombia y la institución con el programa más antiguo de trasplante cardiaco del país, creó el programa de falla cardiaca, como respuesta a la necesidad de un modelo de atención integral que respondiera a los retos de la población con esta enfermedad.

La clínica CardioVID implementó entonces en forma exitosa la primera clínica de falla cardiaca en la ciudad de Medellín, y fue pionera en alternativas innovadoras en cuanto a mejoría de la calidad de vida, la supervivencia y los costos en atención en salud de los pacientes con falla cardiaca. Hoy, después de tres años de funcionamiento, se ha logrado establecer un área extensa de experticia clínica, que le ha permitido involucrar nuevas tecnologías como seguimiento remoto de pacientes, la certificación internacional de parte de Medtronic Heart Failure Solutions and Sanger Cardiovascular Institute - USA y la generación de conocimiento a partir de publicaciones de artículos de investigación en revistas nacionales y la presentación de resultados en congresos internacionales.

En la actualidad cuenta con una población de 800 pacientes en seguimiento activo en quienes se ha demostrado un incremento significativo en la fracción de eyección y mejoría de la clase funcional, así como una disminución de las consultas a urgencias $(29,3 \%)$, las rehospitalizaciones $(66,8 \%)$ y la duración de éstas $(37,5 \%)$, bajar el índice de mortalidad (4\%) y reducir en un $50 \%$ los costos derivados por la atención en hospitalizaciones de la población.

Las más recientes clínicas de falla cardiaca se crearon en la Fundación Santa Fe de Bogotá en agosto del 2014, en cabeza de la Dra. Gina González, médica Internista Cardióloga, especializada en Falla cardiaca del Hospital del Mar en Barcelona bajo la tutela del Dr. Joseph Comin-Collet.

La clínica cuenta con todos los servicios de apoyo de una institución que como la Fundación Santa Fe es un hospital general. Aproximadamente el $90 \%$ de los pacientes se encuentran en entidades de salud a través de servicios complementarios, prepagadas, pólizas, convenios internacionales y particulares. El $10 \%$ pertenece al plan obligatorio de salud (POS).

El equipo multidisciplinario está conformado por una enfermera jefe con entrenamiento en insuficiencia cardiaca, formación realizada antes de la puesta en marcha del proceso por la coordinadora de la clínica de falla (coordinadora Gina González Robledo), con objetivos básicos de aprendizaje bajo la directriz de la Sociedad Europea de Cardiología, para formación de enfermería en insuficiencia cardiaca, y un plan de formación continua que se lleva a cabo mediante actividades académicas programadas de forma mensual. Se cuenta con una médica general entrenada en falla cardiaca en su proceso continuado vinculada en medio tiempo y dos cardiólogos de tiempo parcial, así como un cardiólogo adherente. Durante la fase ambulatoria de la rehabilitación cardiaca, se realiza una consulta con Psicología y Nutrición. La intervención de enfermería refuerza la orientación nutricional con una sesión adicional enfocada en recomendaciones concretas en falla cardiaca.

Se dispone de una línea telefónica para pacientes, habilitada de lunes a viernes de 7 a 5 pm, un correo electrónico y material educativo (librillo diseñado por el grupo con graficadores institucionales que recoge generalidades de falla cardiaca, signos de alarma y medicamentos prohibidos, en el que destacan la importancia y los puntos principales de autocuidado).

Existe un protocolo para realizar seguimientos telefónicos para pacientes que no pueden hacer la intervención presencial por dificultades de movilidad, geográficas o administrativas.

No cuenta aun con el servicio de hospital de día, pero en una nueva edificación del hospital se ha asignado un espacio físico de cuidado paliativo en el cual se contará próximamente con este.

A septiembre de 2015 se realizaron intervenciones a un total de 176 pacientes, $60 \%$ de sexo masculino y $60 \%$ mayores de 70 años, con etiología predominantemente isquémica, fracción de eyección preservada en su mayoría y clase funcional II y III. Se tienen 7 pacientes incluidos con cardiotoxicidad por quimioterapia y 3 pacientes con diagnóstico de amiloidosis cardiaca. Los recursos humanos del programa han aumentado, y hoy día se cuenta con un médico general de tiempo completo, dos cardiólogos de tiempo parcial, una enfermera de tiempo completo y una enfermera en proceso de entrenamiento para vinculación al programa.

Se observa claramente el incremento y desarrollo de los programas de falla cardiaca en Colombia pero estos han llegado a un punto de meseta que ha llevado a evaluar y trabajar nuevas propuestas como la generada por la Dra. Adriana Torres de iniciar y trabajar al interior del Capítulo de falla cardiaca en una estrategia de atención integrada de pacientes con falla cardiaca en tres diferentes niveles de atención lo cual se discutirá ampliamente más adelante en esta misma publicación.

\section{Conflicto de intereses}

Los autores declaran no tener ningún conflicto de intereses. 


\section{Bibliografía}

1. Go AS, Mozaffarian D, Roger VL, Benjamin EJ, Berry JD, Borden W, et al. Heart disease and stroke statistics-2013 update: a report from the American Heart Association. Circulation. 2013; 127:e6.

2. MC Murray JJ, Petrie MC, Murdoch DR, Davie AP. Clinical epidemiology of heart failure: public and private health burden. Eur Heart J. 1998;19 Suppl:P9. 